\title{
Oxymatrine inhibits the proliferation of prostate cancer cells in vitro and in vivo
}

\author{
CUNZAO WU ${ }^{1,2}$, WEIPING HUANG ${ }^{1,2}$, YONG GUO ${ }^{2}$, PENG XIA ${ }^{2}$, \\ XIANBIN SUN ${ }^{2}$, XIAODONG PAN ${ }^{2}$ and WEILIE HU $\mathrm{HU}^{1,3}$ \\ ${ }^{1}$ Graduate School, Southern Medical University, Guangzhou, Guangdong 510515; \\ ${ }^{2}$ Transplantation Center, The First Affiliated Hospital, Wenzhou Medical University, Wenzhou, Zhejiang 325013; \\ ${ }^{3}$ Department of Urology, Guangzhou General Hospital of Guangzhou Military Command, \\ Guangzhou, Guangdong 510010, P.R. China
}

Received May 15, 2014; Accepted January 27, 2015

DOI: $10.3892 / \mathrm{mmr} .2015 .3338$

\begin{abstract}
Oxymatrine is an alkaloid, which is derived from the traditional Chinese herb, Sophora flavescens Aiton. Oxymatrine has been shown to exhibit anti-inflammatory, antiviral, and anticancer properties. The present study aimed to investigate the anticancer effects of oxymatrine in human prostate cancer cells, and the underlying molecular mechanisms of these effects. An MTT assay demonstrated that oxymatrine significantly inhibited the proliferation of prostate cancer cells in a time- and dose-dependent manner. In addition, flow cytometry and a terminal deoxynucleotidyl transferase-mediated dUTP-biotin nick end-labeling assay suggested that oxymatrine treatment may induce prostate cancer cell apoptosis in a dose-dependent manner. Furthermore, western blot analysis demonstrated a significant increase in the expression of p53 and bax, and a significant decrease in that of Bcl-2, in prostrate cancer cells in a dose-dependent manner. In vivo analysis demonstrated that oxymatrine inhibited tumor growth following subcutaneous inoculation of prostate cancer cells into nude mice. The results of the present study suggested that the antitumor properties of oxymatrine, may be associated with the inhibition of cell proliferation, and induction of apoptosis, via the regulation of apoptosis-associated gene expression. Therefore, the results may provide a novel approach for the development of prostate cancer therapy using oxymatrine, which is derived from the traditional Chinese herb, Sophora flavescens.
\end{abstract}

Correspondence to: Professor Weilie Hu, Graduate School, Southern Medical University, 1023 Shatainan Road, Guangzhou, Guangdong 510515, P.R. China

E-mail: weiliehu0515@163.com

Key words: prostate cancer, oxymatrine, proliferation, apoptosis

\section{Introduction}

Prostate cancer is the second most common type of cancer and the sixth leading cause of cancer-related mortality among males worldwide (1). Currently, prostate-specific antigen testing, digital rectal examination and histopathological evaluation of prostate needle biopsies, are performed in order to detect and monitor the progression of prostate cancer $(2,3)$. However, there is increasing evidence to suggest that the resistance of prostate cancer cells to conventional drugs is a significant problem. The investigation of potential novel and effective drugs for the treatment of prostate cancer is therefore required (4).

Traditional Chinese herbs are sources of compounds that may serve as potential therapeutic drugs for cancer (5). $\mathrm{Ku}$ Shen, the dried root of Sophora flavescens Aiton, is a commonly used, traditional Chinese herbal medicine. Oxymatrine, an alkaloid present in $\mathrm{Ku}$ Shen, exhibits anti-inflammatory, anti-allergic, antiviral, antifibrotic and cardiovascular-protective properties (6-8). Furthermore, oxymatrine has been reported to exhibit anticancer properties, such as the inhibition of cancer cell proliferation, the cell cycle and angiogenesis, the promotion of cell apoptosis and reversal of multi-drug resistance in patients with cancer (9-11). A previous study suggested that oxymatrine may suppress angiogenesis by modulating the expression of the NF- $\mathrm{KB}$-mediated vascular endothelial growth factor signaling pathway (12). Furthermore, oxymatrine may induce mitochondria-dependent apoptosis in human osteosarcoma cells by inhibiting the phosphatidylinositol-3 kinase/protein kinase B pathway (13). A number of studies have demonstrated that oxymatrine may inhibit cell growth and the cell cycle, and promote apoptosis in human gastric and breast cancers $(9,14,15)$. However, to the best of our knowledge, the effects of oxymatrine on prostate cancer cells have yet to be investigated. Therefore, the present study aimed to investigate the anticancer effects of oxymatrine on human prostate cancer cells.

\section{Materials and methods}

Reagents. Dulbecco's modified Eagle's medium (DMEM), fetal bovine serum (FBS) and antibiotics (penicillin 
and streptomycin) were purchased from Invitrogen Life Technologies (Carlsbad, CA, USA). Oxymatrine, obtained from Sigma-Aldrich (St. Louis, MO, USA), was dissolved in dimethyl sulfoxide (Sigma-Aldrich) with the stock concentration of $10 \mathrm{mg} / \mathrm{ml}$, and further diluted in the culture medium. Each experiment was repeated at least three times and new dilutions were prepared for each experiment.

Cell culture. DU145 and PC-3 human prostate cancer cell lines and the PNT1B healthy human prostate cell line were obtained from the Chinese Academy of Sciences (Shanghai, China). Cell lines were cultured in DMEM supplemented with $10 \% \mathrm{FBS}, 100 \mathrm{IU} / \mathrm{ml}$ penicillin and $100 \mathrm{mg} / \mathrm{ml}$ streptomycin, and incubated at $37^{\circ} \mathrm{C}$ in $5 \% \mathrm{CO}_{2}$ for $48 \mathrm{~h}$.

Cell proliferation assay. DU145, PC-3 and PNT1B cell lines were seeded into 96 -well plates, incubated overnight and treated with oxymatrine $(0,2,4,6$ and $8 \mathrm{mg} / \mathrm{ml})$. Cell viability was determined using an MTT assay (Sigma-Aldrich). Cells ( $3 \times 10^{4}$ cells/well) were seeded into 96-well plates and incubated overnight at $37^{\circ} \mathrm{C}$ in $5 \% \mathrm{CO}_{2}$. Subsequently, the cells were incubated with different concentrations of oxymatrine $(0,2,4,6$ and $8 \mathrm{mg} / \mathrm{ml})$. MTT $(10 \mathrm{ml} ; 5 \mathrm{mg} / \mathrm{ml})$ was added and the mixture was incubated in darkness at $37^{\circ} \mathrm{C}$ for $2 \mathrm{~h}$. Absorbance was measured at a wavelength of $490 \mathrm{~nm}$ using a microplate reader (FluoDia T70; Photon Technology International, Lawrenceville, NJ, USA).

Flow cytometric analysis. Human prostate cancer cell lines were treated with different concentrations of oxymatrine $(0,4$ and $8 \mathrm{mg} / \mathrm{ml}$ ). Following treatment with oxymatrine for $48 \mathrm{~h}$, cells were trypsinized (Sigma-Aldrich) and centrifuged at $1,000 \mathrm{x} g$ and the pellet was washed twice using PBS. Cells were resuspended and washed with PBS three times. Apoptotic cells were detected using an annexin V-fluorescein isothiocyanate/propidium iodide (annexin V-FITC/IP) cell apoptosis detection kit, according to the manufacturer's instructions (BD Biosciences, Franklin Lakes, NJ, USA).

Western blot analysis. Following oxymatrine treatment, proteins were extracted and separated using a sodium dodecyl sulfate polyacrylamide electrophoresis gel (Bio-Rad Laboratories, Inc., Hercules, CA, USA). Proteins were then transferred to polyvinylidene difluoride membranes (EMD Millipore, Billerica, MA, USA). Membranes were blocked and incubated with the following primary antibodies: Mouse anti-human p53 monoclonal antibody (1:1,000 dilution; cat. no. sc-126), mouse anti-human bcl-2 monoclonal antibody (1:1,000 dilution; cat. no. sc-7382), mouse anti-human bax monoclonal antibody (1:1,000 dilution; cat. no. sc-20067) and mouse anti-human GAPDH monoclonal antibody (1:5,000 dilution; cat. no. sc-365062) (Santa Cruz Biotechnology, Inc., Dallas, TX, USA) overnight at $4^{\circ} \mathrm{C}$. Following washing with Tris-buffered saline and Tween, membranes were incubated with a goat anti-mouse secondary antibody conjugated with horseradish peroxidase $(1: 10,000$ dilution; cat. no. sc-2072; Santa Cruz Biotechnology, Inc.) and visualized using an enhanced chemiluminescent detection reagent from Pierce Biotechnology, Inc. (Rockford, IL, USA).
In vivo xenografts. Approval was obtained from the ethics committee of the First Affiliated Hospital of Wenzhou Medical University (Wenzhou, China). BALB/c homozygous (nu/nu) nude mice (aged 6-8, weeks; weight, 18-20 g), bred in-house, were maintained in a specific pathogen-free environment. PC-3 cells $\left(3 \times 10^{6}\right)$ were suspended in $100 \mu \mathrm{l}$ PBS and subcutaneously injected into the left axilla of recipient mice. On day five, 24 tumor-bearing mice were randomly divided into three groups: The control group was treated with PBS, and two groups were treated with different concentrations of oxymatrine $(50 \mathrm{mg} / \mathrm{kg}$ and $100 \mathrm{mg} / \mathrm{kg}$ body weight). Oxymatrine was administered to the mice, using daily intraperitoneal injections. Tumor volume was calculated using the formula $\mathrm{A} \times \mathrm{B}^{2} \mathrm{x} \pi / 6$, where $\mathrm{A}$ was the length of the longest aspect of the tumor, and $\mathrm{B}$ was the length of the tumor perpendicular to A. Following five weeks of treatment the mice were sacrificed by cervical dislocation and tumor weight was measured.

A terminal deoxynucleotidyl transferase-mediated dUTP-biotin nick end-labeling (TUNEL) assay. Cell apoptosis in mouse tumor samples from six BALB/c mice, was measured in vivo, using a TUNEL assay kit (Roche diagnostics, Indianapolis, IN, USA). Brown nuclei were considered apoptotic. The number of apoptotic cells/1,000 cells was recorded in each field of view, using a microscope (LZ12; Leica Microsystems GmbH, Wetzlar, Germany) at magnification x200.

Statistical analysis. Data are expressed as the mean \pm standard deviation and statistical analysis was carried out using SPSS version 10.0 (SPSS, Inc., Chicago, IL, USA). Comparisons between groups were made using analysis of variance. $\mathrm{P}<0.05$ was considered to indicate a statistically significant difference.

\section{Results}

Oxymatrine inhibits the proliferation of prostate cancer cells. In order to investigate the antiproliferative effects of oxymatrine on prostate cancer cells, DU145 and PNT1B cell lines were treated with different concentrations of oxymatrine $(0,2$, 4, 6 and $8 \mathrm{mg} / \mathrm{ml}$ ) for 24, 48 and $72 \mathrm{~h}$. An MTT assay suggested that oxymatrine significantly inhibited the proliferation of DU145 and PC-3 cell lines in a time- and dose-dependent manner (Fig. 1A and B). By contrast, following treatment with oxymatrine, PNT1B healthy human prostate cell proliferation was not inhibited (Fig. 1C).

Oxymatrine promotes prostate cancer cell apoptosis. Oxymatrine-induced apoptosis in prostate cancer cells was measured using annexin V-FITC/PI double staining. Flow cytometry analysis demonstrated that treatment with oxymatrine resulted in a significant increase in cell apoptosis of PC-3 (Fig. 2A and C) and DU145 (Fig. 2B and D) cell lines, in a dose-dependent manner. These data suggested that oxymatrine treatment may promote prostate cancer cell apoptosis.

Effect of oxymatrine on the expression of apoptosis-related proteins. In order to investigate the possible molecular mechanisms underlying oxymatrine-induced apoptosis of prostate cancer cells, the expression of p53, bax and bcl-2 was analyzed following treatment with different concentrations of 
A

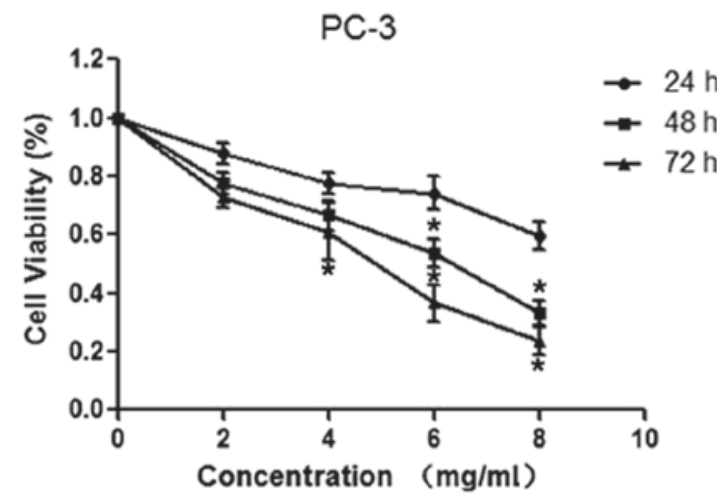

B

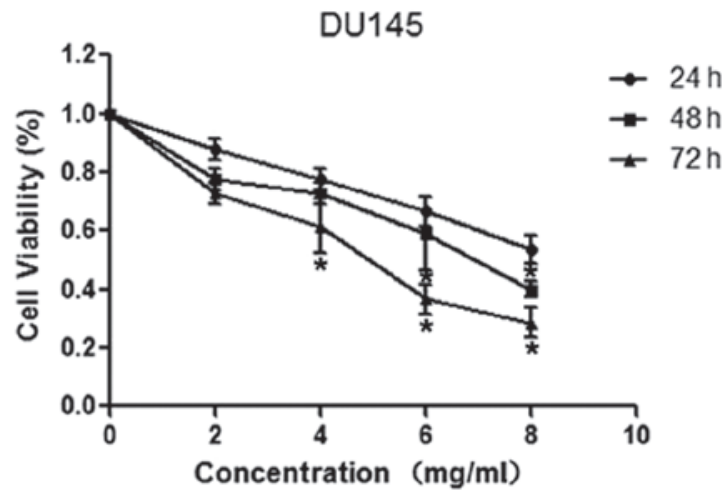

C

PNT1B

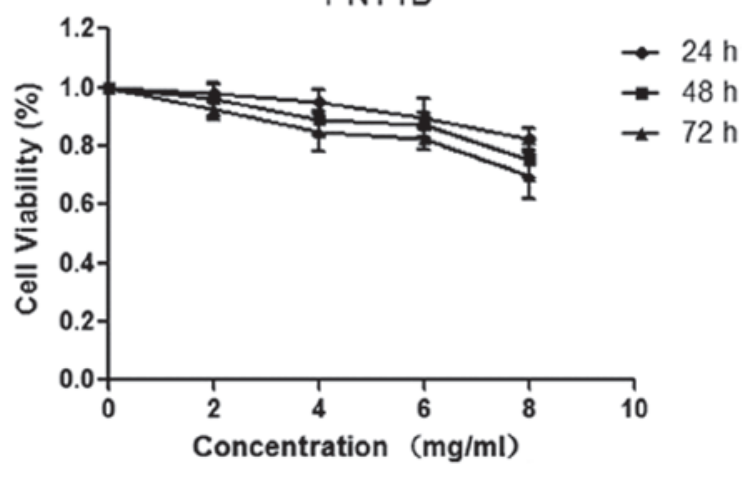

Figure 1. Oxymatrine inhibited the proliferation of prostate cancer cells in vitro. (A) PC-3 and (B) DU145 prostate cancer cells, and (C) PNT1B healthy prostate cells were treated with different concentrations of oxymatrine for 24,48 and $72 \mathrm{~h}$. Cell proliferation was assessed using an MTT assay. Values represent the mean \pm standard deviation of three independent experiments. ${ }^{*} \mathrm{P}<0.05$.

A
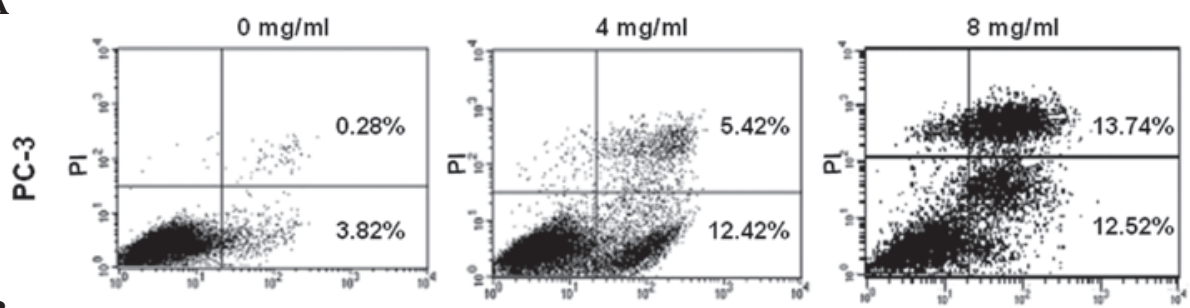

B
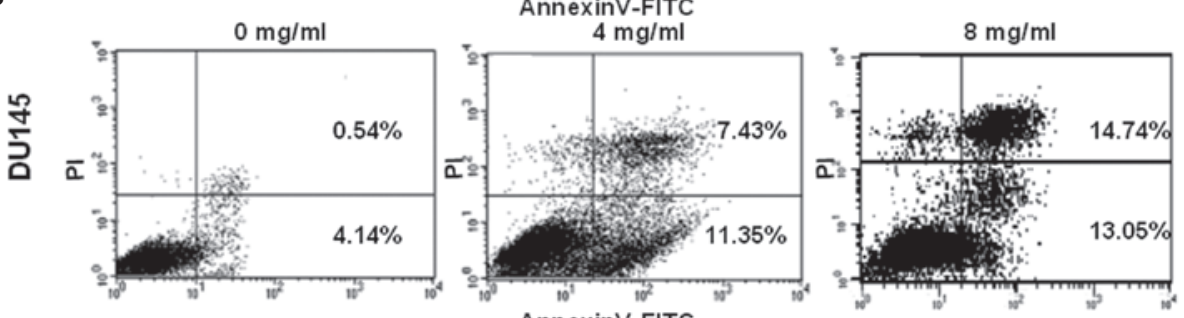

C

PC-3

D

DU145
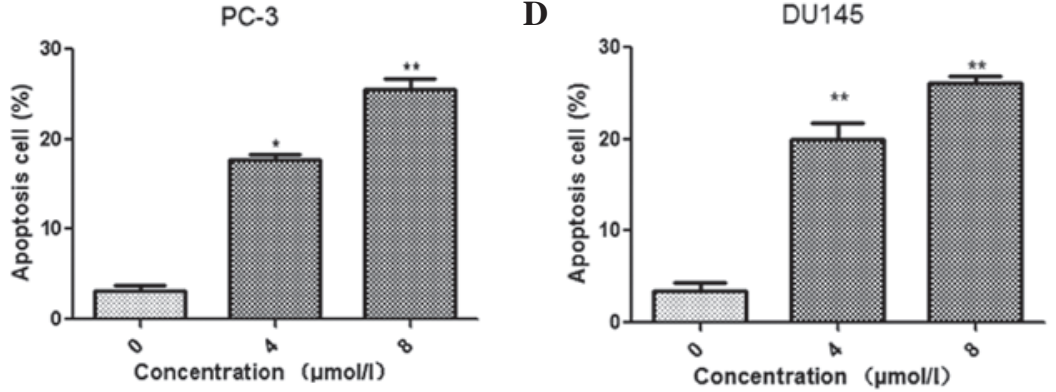

Figure 2. Oxymatrine promoted prostate cancer cell apoptosis. (A) and (C) PC-3, and (B) and (D) DU145 cells were treated with different concentrations of oxymatrine for $48 \mathrm{~h}$. Annexin V-fluorescein isothiocyanate/propidium iodide staining was conducted in order to measure cell apoptosis. Values represent the mean \pm standard deviation of three independent experiments. ${ }^{*} \mathrm{P}<0.05$ and ${ }^{* *} \mathrm{P}<0.01$. 
A

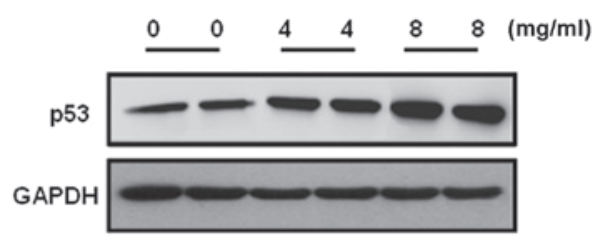

B

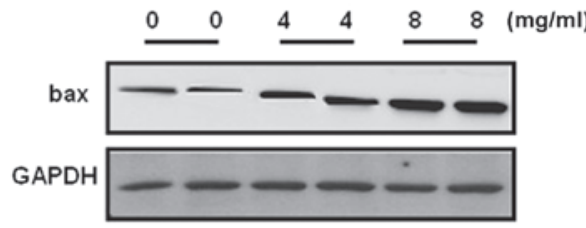

C

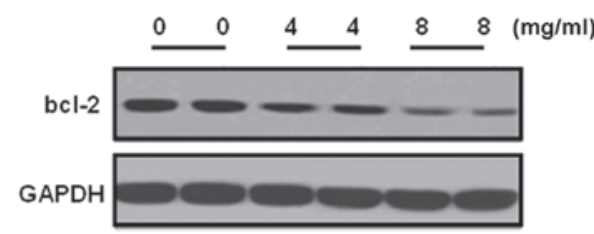

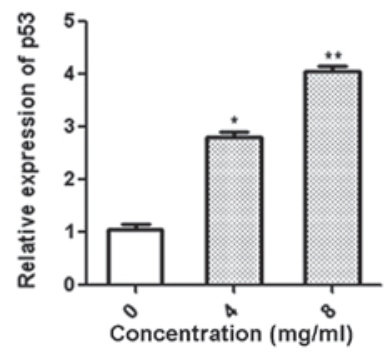
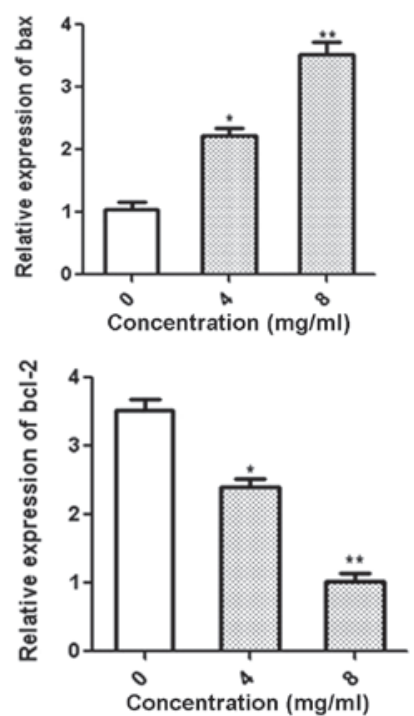

Figure 3. Effect of oxymatrine treatment on apoptosis-associated protein expression in prostate cancer cells. Following treatment with oxymatrine for $48 \mathrm{~h}$, cell lysates were prepared and western blot analysis was performed in order to measure the expression of p53, bax and bcl-2. GAPDH was used as a positive control. Results of representative blots are provided. Relative band intensities were used in order to quantify (A) p53, (B) bax, and (C) bcl-2 protein expression levels. ${ }^{*} \mathrm{P}<0.05$ and ${ }^{* *} \mathrm{P}<0.01$.

A

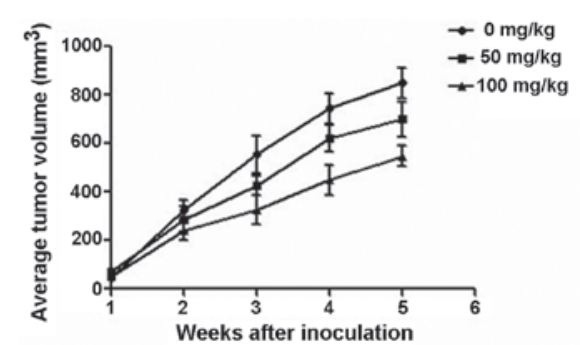

C

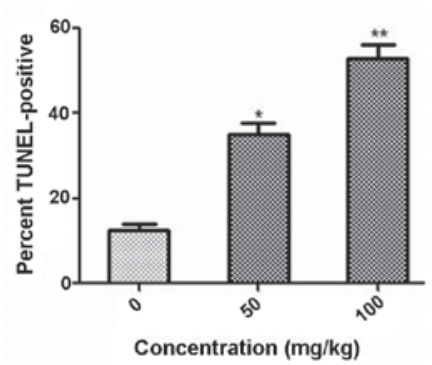

B

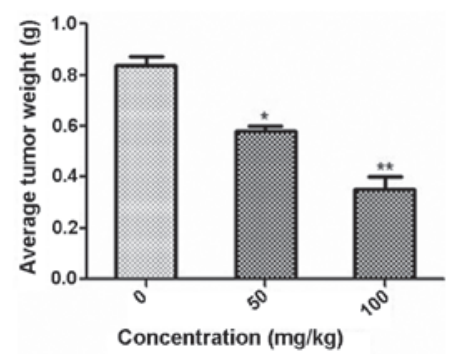

D

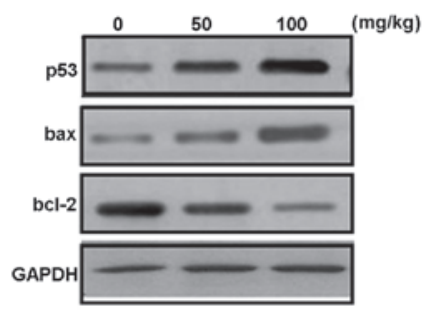

Figure 4. Oxymatrine inhibited PC-3 cell proliferation in vivo. Three concentration levels of oxymatrine $(0,50$ and $100 \mathrm{ml} / \mathrm{kg})$ were intraperitoneally injected into nude mice using PC-3 subcutaneous xenografts. (A) Tumor volume and (B) weight of subcutaneous xenografts were measured at one week intervals. Cell apoptosis in tumor tissues was measured using (C) TUNEL and (D) western blotting of apoptosis-associated proteins, p53, bax and bcl-2. $\mathrm{P}<0.05$ and ${ }^{* *} \mathrm{P}<0.01$. TUNEL, terminal deoxynucleotidyl transferase-mediated dUTP-biotin nick end-labeling.

oxymatrine. Western blotting suggested that the expression of p53 and bcl-2 decreased, whereas that of bax increased, in a dose-dependent manner (Fig. 3).
Oxymatrine reduces prostate cancer cell proliferation in vivo. In order to investigate the effect of oxymatrine on tumor growth in vivo, three concentration levels of 
oxymatrine were intraperitoneally injected into nude mice, using PC-3 subcutaneous xenografts. The results suggested that the volume (Fig. 4A) and weight (Fig. 4B) of tumors in mice significantly decreased in a dose-dependent manner. A TUNEL assay suggested that the number of apoptotic cells increased significantly in a dose-dependent manner (Fig. 4C). In accordance with the in vitro analyses, the expression of apoptosis-associated proteins, p53 and bcl-2 decreased and that of bax increased, in a dose-dependent manner (Fig. 4D). Oxymatrine may therefore reduce prostate cancer cell growth by promoting cell apoptosis in vivo.

\section{Discussion}

Oxymatrine is an alkaloid, which is derived from $\mathrm{Ku}$ Shen and has been shown to be a potential treatment for a number of types of cancers, such as pancreatic (11), gastric (14) and breast cancer (15). However, to the best of our knowledge, the effects of oxymatrine on prostate cancer and the underlying molecular mechanisms of these effects have yet to be investigated. In the present study, oxymatrine treatment was found to promote prostate cancer cell apoptosis and inhibit prostate cancer cell proliferation in vitro and in vivo.

In vitro, an MTT assay demonstrated that oxymatrine treatment significantly inhibited cell proliferation in DU145 and PC-3 prostrate cancer cell lines in a time- and dose-dependent manner. Xenograft tumorigenesis analysis in vivo, demonstrated that following oxymatrine treatment, the weight and size of tumors in PC-3 subcutaneous xenografts were significantly reduced, in a dose-dependent manner. The results of the present study, therefore, indicated that oxymatrine treatment inhibited the proliferation of prostate cancer cells, in vitro and in vivo.

Apoptosis is the process of cell death, characterized by cellular and molecular processes, such as phosphatidylserine externalization, cell shrinkage and chromatin condensation $(16,17)$. Uncontrolled cell proliferation is involved in tumor initiation and progression. Therefore, apoptosis induction provides a potential mechanism for the development of antitumor therapies $(18,19)$. In the present study, oxymatrine treatment induced prostate cancer cell apoptosis in vitro, in a dose-dependent manner, which was demonstrated using flow cytometry and TUNEL analysis.

A number of signalling pathways are involved in the regulation of apoptosis, and numerous molecular markers involved in these pathways have been identified (20-22). For example, p53, encoded by the tumor protein 53 gene is associated with cell apoptosis and cell cycle regulation, in multi-cellular organisms $(21,22)$. Upon internal and external stimuli, such as oxidative stress and viral infection, p53 may activate or suppress a number of downstream target genes involved in apoptosis, such as bax, p53 upregulated modulator of apoptosis and bcl-2 $(23,24)$. Bax is a p53 primary-response gene, involved in a p53-regulated pathway. p53 accumulates in the cytosol and promotes the expression of bax, which permeabilizes mitochondria and promotes cell apoptosis $(25,26)$. The antiapoptotic protein, bcl-2 has been shown to prevent mitochondrial disruption and block cytochrome $c$ release from the mitochondria $(27,28)$. The results of the present study demonstrated that oxymatrine treatment of prostate cancer cells may result in an increase in p53 and bax expression and a decrease in bcl-2 expression, in a dose-dependent manner. Overall, the results suggested that oxymatrine is capable of regulating the expression of apoptosis-associated proteins in prostate cancer cells, in vitro and in vivo.

In conclusion, the results of the present study demonstrated that oxymatrine exhibits antitumor properties in prostate cancer cells, in vitro and in vivo. Furthermore, the results suggested that the antitumor properties of oxymatrine may be attributed to the inhibition of proliferation and the induction of apoptosis via regulation of the expression of apoptosis-associated proteins. Therefore, these findings may provide a novel approach for the development of prostate cancer therapy, using oxymatrine, which is derived from the traditional Chinese herb, Sophora flavescens.

\section{Acknowledgements}

This study was sponsored by Zhejiang Provincial Natural Science Foundation of China (grant no. LY12H10004).

\section{References}

1. Dunn MW and Kazer MW: Prostate cancer overview. Semin Oncol Nurs 27: 241-250, 2011.

2. Katahira K, Takahara T, Kwee TC, Oda S, Suzuki Y, Morishita S, Kitani K, Hamada Y, Kitaoka M and Yamashita Y: Ultra-high-b-value diffusion-weighted MR imaging for the detection of prostate cancer: evaluation in 201 cases with histopathological correlation. Eur Radiol 21: 188-196, 2011.

3. Gosselaar C, Roobol MJ, Roemeling S, van der Kwast TH and Schröder FH: Screening for prostate cancer at low PSA range: the impact of digital rectal examination on tumor incidence and tumor characteristics. Prostate 67: 154-161, 2007.

4. Shi GH, Ye DW, Yao XD, Zhang SL, Dai B, Zhang HL, Shen YJ, Zhu Y, Zhu YP, Xiao WJ and Ma CG: Involvement of microRNA-21 in mediating chemo-resistance to docetaxel in androgen-independent prostate cancer PC3 cells. Acta Pharmacol Sin 31: 867-873, 2010.

5. Liu YH, Li ML, Hsu MY, Pang YY, Chen IL, Chen CK, Tang SW, Lin HY and Lin JY: Effects of a Chinese herbal medicine, Guan-Jen-Huang (Aeginetia indica Linn.), on renal cancer cell growth and metastasis. Evid Based Complement Alternat Med 2012: 935860, 2012.

6. Huang M, Hu YY, Dong XQ, Xu QP, Yu WH and Zhang ZY: The protective role of oxymatrine on neuronal cell apoptosis in the hemorrhagic rat brain. J Ethnopharmacol 143: 228-235, 2012.

7. Chai NL, Fu Q, Shi H, Cai CH, Wan J, Xu SP and Wu BY: Oxymatrine liposome attenuates hepatic fibrosis via targeting hepatic stellate cells. World J Gastroenterol 18: 4199-4206, 2012.

8. Hong-Li S, Lei L, Lei S, Dan Z, De-Li D, Guo-Fen Q, Yan L, Wen-Feng $\mathrm{C}$ and Bao-Feng Y: Cardioprotective effects and underlying mechanisms of oxymatrine against ischemic myocardial injuries of rats. Phytother Res 22: 985-989, 2008.

9. Liu Y, Xu Y, Ji W, Li X, Sun B, Gao Q and Su C: Antitumor activities of matrine and oxymatrine: literature review. Tumour Biol 35: 5111-5119, 2014.

10. Song G, Luo Q, Qin J, Wang L, Shi Y and Sun C: Effects of oxymatrine on proliferation and apoptosis in human hepatoma cells. Colloids Surf B Biointerfaces 48: 1-5, 2006.

11. Ling Q, Xu X, Wei X, Wang W, Zhou B, Wang B and Zheng S: Oxymatrine induces human pancreatic cancer PANC-1 cells apoptosis via regulating expression of Bcl-2 and IAP families, and releasing of cytochrome $c$. J Exp Clin Cancer Res 30: 66, 2011.

12. Chen H, Zhang J, Luo J, Lai F, Wang Z, Tong H, Lu D, Bu H, Zhang $\mathrm{R}$ and Lin S: Antiangiogenic effects of oxymatrine on pancreatic cancer by inhibition of the NF- $\mathrm{B}$-mediated VEGF signaling pathway. Oncol Rep 30: 589-595, 2013.

13. Zhang Y, Sun S, Chen J, Ren P, Hu Y, Cao Z, Sun H and Ding Y: Oxymatrine induces mitochondria dependent apoptosis in human osteosarcoma MNNG/HOS cells through inhibition of PI3K/Akt pathway. Tumour Biol 35: 1619-1625, 2014. 
14. Song MQ, Zhu JS, Chen JL, Wang L, Da W, Zhu L and Zhang WP: Synergistic effect of oxymatrine and angiogenesis inhibitor NM-3 on modulating apoptosis in human gastric cancer cells. World J Gastroenterol 13: 1788-1793, 2007.

15. Zhang Y, Piao B, Zhang Y, Hua B, Hou W, Xu W, Qi X, Zhu X, Pei $\mathrm{Y}$ and Lin H: Oxymatrine diminishes the side population and inhibits the expression of $\beta$-catenin in MCF-7 breast cancer cells. Med Oncol 28 (Suppl 1): S99-S107, 2011.

16. Vogler M, Weber K, Dinsdale D, Schmitz I, Schulze-Osthoff K, Dyer MJ and Cohen GM: Different forms of cell death induced by putative BCL2 inhibitors. Cell Death Differ 16: 1030-1039, 2009.

17. Silva MT: Secondary necrosis: the natural outcome of the complete apoptotic program. FEBS Lett 584: 4491-4499, 2010

18. Frew AJ, Johnstone RW and Bolden JE: Enhancing the apoptotic and therapeutic effects of HDAC inhibitors. Cancer Lett 280 $125-133,2009$

19. Hou J, Wang D, Zhang R and Wang H: Experimental therapy of hepatoma with artemisinin and its derivatives: in vitro and in vivo activity, chemosensitization, and mechanisms of action. Clin Cancer Res 14: 5519-5530, 2008.

20. Vazquez A, Bond EE, Levine AJ and Bond GL: The genetics of the p53 pathway, apoptosis and cancer therapy. Nat Rev Drug Discov 7: 979-987, 2008

21. Hu W, Ge Y, Ojcius DM, Sun D, Dong H, Yang XF and Yan J: p53 signalling controls cell cycle arrest and caspase-independent apoptosis in macrophages infected with pathogenic Leptospira species. Cell Microbiol 15: 1642-1659, 2013.

22. Ben Sahra I, Laurent K, Giuliano S, Larbret F, Ponzio G, Gounon P, Le Marchand-Brustel Y, Giorgetti-Peraldi S, Cormont M, Bertolotto C, et al: Targeting cancer cell metabolism: the combination of metformin and 2-deoxyglucose induces p53-dependent apoptosis in prostate cancer cells. Cancer Res 70: 2465-2475, 2010.
23. Gomez-Lazaro M, Galindo MF, Concannon CG, Segura MF, Fernandez-Gomez FJ, Llecha N, Comella JX, Prehn JH and Jordan J: 6-Hydroxydopamine activates the mitochondrial apoptosis pathway through p38 MAPK-mediated, p53-independent activation of Bax and PUMA. J Neurochem 104 1599-1612, 2008

24. Ramaiah MJ, Pushpavalli SN, Lavanya A, Bhadra K, Haritha V, Patel N, Tamboli JR, Kamal A, Bhadra U and Pal-Bhadra M: Novel anthranilamide-pyrazolo[1,5-a]pyrimidine conjugates modulate the expression of $\mathrm{p} 53-\mathrm{MYCN}$ associated micro RNAs in neuroblastoma cells and cause cell cycle arrest and apoptosis. Bioorg Med Chem Lett 23: 5699-5706, 2013.

25. Deng Y and Wu X: Peg3/Pw1 promotes p53-mediated apoptosis by inducing Bax translocation from cytosol to mitochondria. Proc Natl Acad Sci USA 97: 12050-12055, 2000.

26. Gogada R, Prabhu V, Amadori M, Scott R, Hashmi S and Chandra D: Resveratrol induces p53-independent, X-linked inhibitor of apoptosis protein (XIAP)-mediated Bax protein oligomerization on mitochondria to initiate cytochrome C release and caspase activation. J Biol Chem 286: 28749-28760, 2011.

27. Bishayee K, Chakraborty D, Ghosh S, Boujedaini N and Khuda-Bukhsh AR: Lycopodine triggers apoptosis by modulating 5-lipoxygenase, and depolarizing mitochondrial membrane potential in androgen sensitive and refractory prostate cancer cells without modulating p53 activity: signaling cascade and drug-DNA interaction. Eur J Pharmacol 698: 110-121, 2013.

28. Liu Y, Yang Y, Ye YC, Shi QF, Chai K, Tashiro S, Onodera S and Ikejima T: Activation of ERK-p53 and ERK-mediated phosphorylation of Bcl-2 are involved in autophagic cell death induced by the c-Met inhibitor SU11274 in human lung cancer A549 cells. J Pharmacol Sci 118: 423-432, 2012. 